Diagonal Actions and Borel Equivalence Relations

Author(s): Longyun Ding and Su Gao

Source: The Journal of Symbolic Logic, Vol. 71, No. 4 (Dec., 2006), pp. 1081-1096

Published by: Association for Symbolic Logic

Stable URL: https://www.jstor.org/stable/27588503

Accessed: 18-03-2021 00:58 UTC

JSTOR is a not-for-profit service that helps scholars, researchers, and students discover, use, and build upon a wide range of content in a trusted digital archive. We use information technology and tools to increase productivity and facilitate new forms of scholarship. For more information about JSTOR, please contact support@jstor.org.

Your use of the JSTOR archive indicates your acceptance of the Terms \& Conditions of Use, available at https://about.jstor.org/terms access to The Journal of Symbolic Logic 


\title{
DIAGONAL ACTIONS AND BOREL EQUIVALENCE RELATIONS
}

\author{
LONGYUN DING AND SU GAO
}

\begin{abstract}
We investigate diagonal actions of Polish groups and the related intersection operator on closed subgroups of the acting group. The Borelness of the diagonal orbit equivalence relation is characterized and is shown to be connected with the Borelness of the intersection operator. We also consider relatively tame Polish groups and give a characterization of them in the class of countable products of countable abelian groups. Finally an example of a logic action is considered and its complexity in the Borel reducbility hierarchy determined.
\end{abstract}

§1. Introduction. We are interested in the following question in the descriptive set theory of Polish group actions:

Let $G$ be a Polish group and $X, Y$ be Borel $G$-spaces with the orbit equivalence relations $E_{G}^{X}$ and $E_{G}^{Y}$ Borel. Consider the diagonal action of $G$ on $X \times Y$ :

$$
g \cdot(x, y)=(g \cdot x, g \cdot y)
$$

When is $E_{G}^{X \times Y}$ Borel?

The question appeals to us for various reasons. On the one hand, it is fundamental and simple, but the solution seems to be non-trivial. On the other hand, it has potential applications to important classification problems such as that of bounded linear operators of the separable Hilbert space. In fact, if we denote by $U_{\infty}$ the group of all unitary operators on a separable Hilbert space, it is well known that the classification problem for all bounded linear operators is equivalent to finding the complexity for the orbit equivalence relation of the diagonal conjugacy action of $U_{\infty}$ on $U_{\infty} \times U_{\infty}$ (c.f., e.g., Introduction of [7]). The Borelness of this equivalence relation is still open.

Borelness of orbit equivalence relations has been one of the main focuses of investigation in the descriptive set theory of Polish group actions (c.f. $[6,1,8]$ ). One of the first characterizations used a uniform bound on the Borel complexity of orbits (when the space is Polish) and was due to Sami.

TheOREM 1.1 (Sami [6]). Let $G$ be a Polish group acting in a Borel manner on a Polish space $X$. Then $E_{G}^{X}$ is Borel iff there is $\alpha<\omega_{1}$ such that every orbit is $\underset{\sim}{\boldsymbol{\Pi}_{\alpha}^{0}}$.

Becker and Kechris considered the stabilizer groups of the actions and characterized the Borelness of the orbit equivalence relation by the stabilizer map.

Received February 24, 2005.

2000 Mathematics Subject Classification. Primary 04A15, 54H05, 22 F05.

The second author's research was supported by the U.S. NSF grant DMS-0501039.

(C) 2006, Association for Symbolic Logic $0022-4812 / 06 / 7104-0001 / \$ 2.60$ 
TheOREM 1.2 (Becker-Kechris [1]). Let $G$ be a Polish group and X a Borel G-space. Then the following are equivalent:

(i) $E_{G}^{X}$ is Borel.

(ii) The map

$$
\begin{aligned}
X & \rightarrow \mathscr{F}(G) \\
x & \mapsto G_{x}=\{g \in G: g \cdot x=x\}
\end{aligned}
$$

is Borel.

(iii) The map

$$
\begin{aligned}
X \times X & \rightarrow \mathscr{F}(G) \\
(x, u) & \mapsto G_{x, u}=\{g \in G: g \cdot x=u\}
\end{aligned}
$$

is Borel.

Both these theorems will play a central role in our study below. For the diagonal action of $G$ on $X \times Y$, the stabilizer is simply

$$
G_{(x, y)}=G_{x} \cap G_{y} .
$$

Thus to guarantee Borelness of $E_{G}^{X \times Y}$ under our hypothesis that $E_{G}^{X}$ and $E_{G}^{Y}$ are Borel, an immediate sufficient condition is the Borelness of the intersection operator on $\mathscr{F}(G)$.

In this article we give some characterizations for the Borelness of $E_{G}^{X \times Y}$ and also for the Borelness of the intersection operator on closed subgroups of $G$. We call a group $G$ relatively tame if the intersection operator on its closed subgroups is Borel. Then we will investigate the notion of relative tameness and characterize them completely within a class of Polish groups. In fact, the class of Polish groups we will consider is that of all countable products of countable abelian groups, as considered in [8]. In [8] Solecki has characterized the tame groups within this class. We will show that a group in this class is relatively tame if and only if it is tame.

The article is organized as follows. In section 2 we recall some basic notation and define some other notation to be used throughout the rest of the article. In section 3 we give characterizations for the Borelness of the diagonal orbit equivalence relation. In section 4 we give the characterizations for the Borelness of the intersection operator. In section 5 we consider countable products of countable abelian groups and characterize relative tame groups of this form. In section 6 we consider some specific examples of diagonal actions by the permuation group $S_{\infty}$ and completely characterize the complexity of these equivalence relations.

Acknowledgement. We thank G. Hjorth and A. S. Kechris for helpful discussions on the subject of this article. This article was written during the first author's visit to the University of North Texas. He would like to thank UNT for the hospitality.

§2. Basic notation. Let $G$ be a Polish group and $X$ a Polish or standard Borel space. An action of $G$ on $X$ is a function

$$
a: G \times X \rightarrow X
$$

satisfying, for all $g, h \in G$ and $x \in X, a(g, a(h, x))=a(g h, x)$ and $a\left(1_{G}, x\right)=x$, where $1_{G}$ is the identity element of $G$. We say that $G$ acts on $X$ in a Borel manner or that the action is Borel if $a$ is a Borel function. In case $X$ is a standard Borel space 
on which $G$ acts in a Borel manner, we say that $X$ is a Borel $G$-space. When there is no danger of ambiguity we also write $g \cdot x$ for $a(g, x)$.

Let $X$ be a Borel $G$-space. The orbit equivalence relation, denoted by $E_{G}^{X}$, is an equivalence relation on $X$ defined by

$$
x_{1} E_{G}^{X} x_{2} \Longleftrightarrow \exists g \in G\left(g \cdot x_{1}=x_{2}\right)
$$

for $x_{1}, x_{2} \in X$. When there is no danger of confusion we will write $E^{X}$ instead of $E_{G}^{X}$, especially when the acting group $G$ is uniquely determined from the context.

If $X$ and $Y$ are both Borel $G$-spaces, then the diagonal action of $G$ on $X \times Y$ is defined by

$$
g \cdot(x, y)=(g \cdot x, g \cdot y)
$$

for $x \in X$ and $y \in Y$. Unless we specify otherwise this will be the standard action of $G$ on the product space $X \times Y$. We also follow a similar convention for products with more than two factors.

If $X$ is a Polish space the Effros Borel space $\mathscr{F}(X)$ is defined as the space of all closed subsets of $X$ with the Borel structure generated by sets of the form

$$
\{F \in \mathscr{F}(X): F \cap U \neq \emptyset\}
$$

for $U$ an open subset of $X$. Basic properties of the Effros Borel space can be found in [4] §12.C. Here we only recall that $\mathscr{F}(X)$ is a standard Borel space and the following selection theorem due to Kuratowski-Ryll-Nardzewski.

THEOREM 2.1 (Kuratowski-Ryll-Nardzewski). Let $X$ be a Polish space. Then there is a Borel function $s: \mathscr{F}(X) \rightarrow X$ such that for non-empty $F \in \mathscr{F}(X), s(F) \in F$.

We call the above function $s$ a Borel selector for $\mathscr{F}(X)$.

In fact we will be mostly considering the Effros Borel space $\mathscr{F}(G)$ for a Polish group $G$. In this context we also define the following subclasses of $\mathscr{F}(G)$. Let $\mathscr{G}(G)$ denote the collection of all closed subgroups of $G$. Then $\mathscr{G}(G)$ is a Borel subset of $\mathscr{F}(G)$. To see this, let $\mathscr{B}$ be a countable basis for the topology of $G$. Then for $F \in \mathscr{F}(G), F \in \mathscr{G}(G)$ iff

$$
\begin{gathered}
\forall U \in \mathscr{B}\left(1_{G} \in U \Rightarrow F \cap U \neq \emptyset\right) \wedge \forall U \in \mathscr{B}\left(F \cap U \neq \emptyset \Leftrightarrow F \cap U^{-1} \neq \emptyset\right) \\
\wedge \forall U, V \in \mathscr{B}[(F \cap U \neq \emptyset \wedge F \cap V \neq \emptyset) \rightarrow F \cap U V \neq \emptyset] .
\end{gathered}
$$

Thus $\mathscr{G}(G)$ is a standard Borel space with the Borel structure inherited from that of $\mathscr{F}(G)$.

Let $\mathscr{C}(G)$ denote the collection of all cosets of closed subgroups of $G$, i.e., sets of the form $g H$ where $g \in G$ and $H \in \mathscr{G}(G)$. To see that $\mathscr{C}(G)$ is also a Borel subset of $\mathscr{F}(G)$, we recall the following theorem of Dixmier (c.f. [1] Theorem 1.2.4).

Theorem 2.2 (Dixmier). Let $G$ be a Polish group. Then there is a Borel set $T^{l} \subset$ $G \times \mathscr{G}(G)$ such that for any $H \in \mathscr{G}(G), T_{H}^{l}=\left\{g \in G:(g, H) \in T^{l}\right\}$ is a transversal for the left-cosets of $H$, i.e., $T_{H}^{l}$ contains exactly one element of each left-coset of $H$.

Now the map $\sigma: T^{l} \rightarrow \mathscr{F}(G)$ defined by $\sigma(g, H)=g H$ is a Borel injection with range $\mathscr{C}(G)$. Hence $\mathscr{C}(G)$ is Borel in $\mathscr{F}(G)$ and also a standard Borel space with the inherited Borel structure.

We remark that Dixmier's theorem can also be used to obtain a Borel set $T^{r}$ for the right-cosets of closed subgroups in $G$. 
Let $G$ be a Polish group and $X$ be a Borel $G$-space. For each $x \in X$, the stabilizer is defined as

$$
G_{x}=\{g \in G: g \cdot x=x\} .
$$

Each $G_{x}$ is a closed subgroup of $G$. The stabilizer map is the map $x \mapsto G_{x}$ from $X$ into $\mathscr{G}(G)$. Part (ii) of Becker-Kechris theorem states that $E_{G}^{X}$ is Borel iff the stabilizer map is Borel. For $x, u \in X$, we also denote

$$
G_{x, u}=\{g \in G: g \cdot x=u\} .
$$

Each $G_{x, u}$ is an element of $\mathscr{C}(G)$. We will use the following notation:

$$
\mathcal{S}^{X}(G)=\left\{G_{x}: x \in X\right\} \text { and } \mathscr{T}^{X}(G)=\left\{G_{x, u}: x, u \in X\right\} .
$$

In general $\mathcal{S}^{X}(G)$ is a $\underset{\sim}{\Sigma_{1}^{1}}$ subset of $\mathscr{G}(G)$ and $\mathscr{T}^{X}(G)$ is a $\underset{\sim}{\Sigma_{1}^{1}}$ subset of $\mathscr{C}(G)$. The following diagram summarizes their relationship:

$$
\begin{array}{ccc}
\mathcal{S}^{X}(G) & \subseteq \mathscr{G}(G) \leqq \\
\cap & \cap \\
\mathscr{T}^{X}(G) & \subseteq \mathscr{C}(G) \subseteq
\end{array}
$$

A final piece of notation: we let $\mathscr{N}$ denote the Baire space $\mathbb{N}^{\mathbb{N}}$.

§3. Products of stabilizers. In this section we consider the diagonal action of $G$ on a product $X \times Y$ and characterize the Borelness of the orbit equivalence relation on an invariant Borel subset $Z \subseteq X \times Y$.

TheOREM 3.1. Let $G$ be a Polish group, $X$ and $Y$ be Borel $G$-spaces with $E^{X}$ and $E^{Y}$ Borel, and $Z \subseteq X \times Y$ an invariant Borel subset. Then the following are equivalent:

(i) $E^{Z}$ is Borel.

(ii) $\left\{(g h, x, y): g \in G_{x}, h \in G_{y},(x, y) \in Z\right\}$ is Borel.

(iii) There is $\alpha<\omega_{1}$ so that for any $(x, y) \in Z, G_{x} G_{y}$ is $\underset{\sim}{\mathbf{\Pi}_{\alpha}^{0}}$.

Proof. (i) $\Rightarrow$ (ii). By the Becker-Kechris theorem cited in the Introduction, the maps $x \mapsto G_{x}$ from $X$ to $\mathscr{F}(G), y \mapsto G_{y}$ from $Y$ to $\mathscr{F}(G)$ and $(x, y) \mapsto G_{(x, y)}$ from $Z$ to $\mathscr{F}(G)$ are Borel. Let $T^{l}$ and $T^{r}$ be Borel subsets of $G \times \mathscr{G}(G)$ giving Borel transversals for left- and right-cosets, respectively, by Dixmier's theorem. Now define $D \subseteq G^{3} \times X \times Y$ as follows:

$$
\begin{aligned}
(g, h, k, x, y) \in D \Leftrightarrow & (x, y) \in Z \wedge k \in G_{(x, y)} \wedge g \in G_{x} \wedge\left(g, G_{(x, y)}\right) \in T^{l} \\
& \wedge h \in G_{y} \wedge\left(h, G_{(x, y)}\right) \in T^{r} .
\end{aligned}
$$

Then let $f: D \rightarrow G \times X \times Y$ be

$$
f(g, h, k, x, y)=(g k h, x, y) .
$$

Then it is easy to check that $f$ is continuous and one-to-one. Note that the range of $f$ is exactly $\left\{(g h, x, y): g \in G_{x}, h \in G_{y},(x, y) \in Z\right\}$, which is Borel being an injective image of a Borel map.

(ii) $\Rightarrow$ (iii) is trivial.

(iii) $\Rightarrow$ (i). Again by the Becker-Kechris theorem, the maps $(x, u) \mapsto G_{x, u}$ from $X^{2}$ into $\mathscr{F}(G)$ and $(y, v) \mapsto G_{y, v}$ from $Y^{2}$ to $\mathscr{F}(G)$ are Borel. Let $s$ be a Borel 
selector of $\mathscr{F}(G)$. Define $\tau_{X}(x, u)=s\left(G_{x, u}\right)$ for $x, u \in X$. Then $\tau_{X}$ is Borel and $G_{x, u}=\tau_{X}(x, u) G_{x}$. Similarly define $\tau_{Y}(y, v)=s\left(G_{y, v}\right)$ for $y, v \in Y$.

Now for any $x, u \in X, y, v \in Y$ we have

$$
\begin{aligned}
G \cdot(x, y)=G \cdot(u, v) & \Longleftrightarrow \exists g \in G(g \cdot x=u \wedge g \cdot y=v) \\
& \Longleftrightarrow G_{x, u} \cap G_{y, v} \neq \emptyset \\
& \Longleftrightarrow \tau_{X}(x, u) G_{x} \cap \tau_{Y}(y, v) G_{y} \neq \emptyset \\
& \Longleftrightarrow \tau_{X}(x, u)^{-1} \tau_{Y}(y, v) \in G_{x} G_{y} .
\end{aligned}
$$

Now fix Polish topologies on $X, Y$ and $Z$ so that the topology on $Z$ is finer than the subspace topology on it inherited from the product space $X \times Y$ (c.f., e.g., Theorem 13.1 in [4]). Assume that $\tau_{X}$ and $\tau_{Y}$ are of Baire class $\xi$ for $\xi<\omega_{1}$. Then for any $(x, y) \in Z$, the orbit is characterized as

$$
G \cdot(x, y)=\left\{(u, v): \tau_{X}(x, u)^{-1} \tau_{Y}(y, v) \in G_{x} G_{y}\right\}
$$

and is $\prod_{\sim+\alpha}^{0}$ by our assumption. By Sami's theorem $E_{Z}$ is Borel.

Clause (iii) of this theorem is particularly interesting: it reduces the question of Borelness of the diagonal orbit equivalence relation to questions about the collection $\mathcal{S}^{X}(G)$. The following corollary is immediate.

Corollary 3.2. Let $X_{1}, X_{2}, Y_{1}, Y_{2}$ be Borel $G$-spaces with $E^{X_{1}}, E^{X_{2}}, E^{Y_{1}}, E^{Y_{2}}$ all Borel. Assume that $\mathcal{S}^{X_{1}}(G) \subseteq \mathcal{S}^{X_{2}}(G), \mathcal{S}^{Y_{1}}(G) \subseteq \mathcal{S}^{Y_{2}}(G)$. If $E^{X_{2} \times Y_{2}}$ is Borel, so is $E^{X_{1} \times Y_{1}}$.

A curious question at this point is whether there is a version of Theorem 3.1 for finite products with more than two factors. We do not know the answer.

Next we characterize subsets of $\mathscr{G}(G)$ that can arise as $\mathcal{S}^{X}(G)$ for some Borel $G$-space $X$ on which $E^{X}$ is Borel. As we remarked before, $\mathcal{S}^{X}(G)$ is in general $\underset{\sim}{\Sigma_{1}^{1}}$. Recall that a subset $\mathcal{S} \subseteq \mathscr{G}(G)$ is said to be closed under conjugation if for $g \in G$ and $H \in \mathcal{S}$ we have $g \mathrm{Hg}^{-1} \in \mathcal{S} . \mathcal{S}^{X}(G)$ is always closed under conjugation.

THEOREM 3.3. Let $\mathcal{S} \subseteq \mathscr{G}(G)$. Then the following are equivalent:

(i) There is a Borel G-space $X$ on which $E^{X}$ is Borel such that $\mathcal{S}=\mathcal{S}^{X}(G)$.

(ii) $\mathcal{S}$ is $\Sigma_{\sim}^{1}$ and closed under conjugation.

Proof. (i) $\Rightarrow$ (ii) is trivial.

(ii) $\Rightarrow($ i). Let $\varphi: \mathscr{N} \rightarrow \mathscr{G}(G)$ be a Borel map with $\mathcal{S}=\varphi(\mathcal{N})$. Let $s$ be a Borel selector of $\mathscr{F}(G)$. Define

$$
X=\{(k, x) \in G \times \mathscr{N}: k=s(k \varphi(x))\} .
$$

Then $X$ is a Borel subset of $G \times \mathscr{N}$, and hence is a standard Borel space. Define an action of $G$ on $X$ as follows:

$$
g \cdot(k, x)=(s(g k \varphi(x)), x) .
$$

It is straightforward to check that this is a Borel action on $X$. Note that

$$
\begin{aligned}
g \in G_{(k, x)} & \Longleftrightarrow s(g k \varphi(x))=k=s(k \varphi(x)) \\
& \Longleftrightarrow g k \varphi(x)=k \varphi(x) \\
& \Longleftrightarrow g \in k \varphi(x) k^{-1} .
\end{aligned}
$$


Thus $G_{(k, x)}=k \varphi(x) k^{-1} \in \mathcal{S}$ and the map $(k, x) \mapsto G_{(k, x)}$ is Borel. It follows that $E^{X}$ is Borel and that $\mathcal{S}=\mathcal{S}^{X}(G)$.

§4. The intersection operator. In this section we give various characterizations for the Borelness of the intersection operator on $\mathscr{G}(G)$ and other related classes. It turns out that one of the characterizations is the Borelness of a particular diagonal orbit equivalence relation.

Recall that $\mathscr{C}(G)$ is the collection of all (left-) cosets of closed subgroups of $G$, i.e.,

$$
\mathscr{C}(G)=\{g H: g \in G, H \in \mathscr{G}(G)\} .
$$

$\mathscr{C}(G)$ is a Borel $G$-space with the natural action $k \cdot g H=k g H$. Note that $E^{\mathscr{C}}(G)$ is Borel, since it is in fact smooth with a Borel transversal $\mathscr{G}(G)$. We will make use of the diagonal action of $G$ on $\mathscr{C}(G)^{2}$.

THEOREM 4.1. Let $G$ be a Polish group. Then the following are equivalent:

(i) $E^{\mathscr{E}(G)^{2}}$ is Borel.

(ii) The map $(H, K) \mapsto H \cap K$ from $\mathscr{G}(G)^{2}$ to $\mathscr{G}(G)$ is Borel.

(iii) For any Borel $G$-spaces $X$ and $Y$ with $E^{X}$ and $E^{Y}$ Borel, $E^{X \times Y}$ is Borel.

(iv) There is $\alpha<\omega_{1}$ so that for any $H, K \in \mathscr{G}(G), H K$ is $\underset{\sim}{\boldsymbol{\Pi}_{\alpha}^{0}}$.

Proof. (i) $\Rightarrow$ (ii). If $E^{\mathscr{C}(G)^{2}}$ is Borel then the map $(h H, k K) \mapsto G_{(h H, k K)}=G_{h H} \cap$ $G_{k K}$ from $\mathscr{C}(G)^{2}$ into $\mathscr{G}(G)$ is Borel, and therefore its restriction on $\mathscr{G}(G)^{2}:(H, K) \mapsto$ $G_{H} \cap G_{K}=H \cap K$ is Borel.

(ii) $\Rightarrow$ (iii) and (iii) $\Rightarrow$ (i) are trivial.

(i) $\Rightarrow$ (iv) and (iv) $\Rightarrow$ (iii) follow from Theorem 3.1.

Note that the clauses in the above theorem are also equivalent to the following statement:

(iii) $^{\prime}$ For any Borel $G$-spaces $X_{1}, \ldots, X_{n}$ with $E^{X_{i}}$ Borel for all $i \leq n$, $E^{X_{1} \times \cdots \times X_{n}}$ is Borel.

We say that a Polish group $G$ is relatively tame if it satisfies one of the equivalent conditions in Theorem 4.1. Recall that a Polish group is tame if $E^{X}$ is Borel for any Borel $G$-space $X$. If $G$ is tame then it is relatively tame. Also it is easy to see that if $G$ is relatively tame then so are all of its closed subgroups and topological factor groups.

We now turn to Borelness of the intersection operator on other, more general subclasses of $\mathscr{G}(G)^{2}$. Some of these classes we consider will be $\underset{\sim}{\Sigma_{1}^{1}}$ and non-Borel. For them we need the following curious fact.

Lemma 4.2. Let $X, Y$ and $Z$ be standard Borel spaces, $A \subseteq Y$ be $\underset{\sim}{\Sigma_{1}^{1}}, f: A \rightarrow Z$, and $\phi: X \rightarrow Y$ be a Borel function with $\phi(X)=A$. If $f \circ \phi$ is Borel, then $f$ is Borel.

Proof. For $B$ Borel in $Z$, we will show that $f^{-1}(B)$ is Borel in $A$. Let $C=$ $\phi^{-1}\left(f^{-1}(B)\right)$, then $C$ is Borel in $X$. Then both $f^{-1}(B)=\phi(C)$ and $A \backslash f^{-1}(B)=$ $\phi(X \backslash C)$ are $\Sigma_{1}^{1}$ in $Y$. By Lusin Separation Theorem, there is a Borel set $D \subseteq Y$ such that $f^{-1}(B) \subseteq D$ and $\left(A \backslash f^{-1}(B)\right) \cap D=\emptyset$. This means $f^{-1}(B)=A \cap D$ is Borel in $A$.

The following corollary is immediate from the above lemma. 
Corollary 4.3. Let $X$ and $Y$ be standard Borel spaces and $f: X \rightarrow Y$. Then the following are equivalent:

(i) $f$ is Borel.

(ii) For some standard Borel space $Z$ and Borel surjection $\phi: Z \rightarrow X, f \circ \phi$ is Borel.

(iii) For every standard Borel space $Z$ and Borel surjection $\phi: Z \rightarrow X, f \circ \phi$ is Borel.

THEOREM 4.4. Let $\mathcal{S}_{1}$ and $\mathcal{S}_{2}$ be ${\underset{\sim}{1}}_{1}^{1}$ subsets of $\mathscr{G}(G)$ both closed under conjugation. Then the following are equivalent:

(i) The map $(H, K) \mapsto H \cap K$ from $\mathcal{S}_{1} \times \mathcal{S}_{2}$ to $\mathscr{G}(G)$ is Borel.

(ii) For any Borel $G$-spaces $X$ and $Y$ with $E^{X}$ and $E^{Y}$ Borel, if $\mathcal{S}^{X}(G) \subseteq \mathcal{S}_{1}$ and $\mathcal{S}^{Y}(G) \subseteq \mathcal{S}_{2}$, then $E^{X \times Y}$ is Borel.

(iii) There are Borel $G$-spaces $X$ and $Y$ such that $\mathcal{S}^{X}(G)=\mathcal{S}_{1}, \mathcal{S}^{Y}(G)=\mathcal{S}_{2}$ and $E^{X}, E^{Y}$ and $E^{X \times Y}$ are Borel.

(iv) There is $\alpha<\omega_{1}$ such that for any $H \in \mathcal{S}_{1}$ and $K \in \mathcal{S}_{2}, H K \in \underset{\sim}{\boldsymbol{\Pi}} \mathbf{0}$.

Proof. (i) $\Rightarrow$ (ii) is trivial.

(ii) $\Rightarrow$ (iii). By Theorem 3.3 there are Borel $G$-spaces $X$ and $Y$ with $E^{X}$ and $E^{Y}$ Borel such that $\mathcal{S}^{X}(G)=\mathcal{S}_{1}$ and $\mathcal{S}^{Y}(G)=\mathcal{S}_{2}$. By (ii) $E^{X \times Y}$ is also Borel.

(iii) $\Rightarrow$ (i). Denote the map $(H, K) \mapsto H \cap K$ from $\mathcal{S}_{1} \times \mathcal{S}_{2}$ into $\mathscr{G}(G)$ by $f$. Let the Borel $G$-spaces $X$ and $Y$ be given. Define $\phi: X \times Y \rightarrow \mathscr{G}(G)^{2}$ by $\phi(x, y)=$ $\left(G_{x}, G_{y}\right)$. Then $\phi$ is Borel and also the map $(x, y) \mapsto G_{(x, y)}=G_{x} \cap G_{y}$, which is exactly $f \circ \phi$, is Borel. By Lemma 4.2, $f$ is Borel.

(iii) $\Rightarrow$ (iv) and (iv) $\Rightarrow$ (ii) follow from Theorem 3.1.

Next we consider even more general subclasses of $\mathscr{G}(G)^{2}$. For $Z$ an invariant Borel subset of $X \times Y$, we let

$$
\mathscr{D}^{Z}(G)=\left\{\left(G_{x}, G_{y}\right):(x, y) \in Z\right\} .
$$

The following is a strengthening of Corollary 3.2 but it also follows immediately from Theorem 3.1.

Corollary 4.5. Let $X_{1}, X_{2}, Y_{1}, Y_{2}$ be Borel $G$-spaces with $E^{X_{1}}, E^{X_{2}}, E^{Y_{1}}, E^{Y_{2}}$ Borel. Let $Z_{i}$ be an invariant Borel subset of $X_{i} \times Y_{i}$ for $i=1,2$. Assume that $\mathscr{D}^{Z_{1}}(G) \subseteq \mathscr{D}^{Z_{2}}(G)$. If $E^{Z_{2}}$ is Borel, so is $E^{Z_{1}}$.

We say that a subset $\mathscr{D} \subseteq \mathscr{G}(G)^{2}$ is closed under conjugation if for any $g \in G$ and $(H, K) \in \mathscr{D}$, we have $\left(g \mathrm{Hg}^{-1}, g \mathrm{Kg}^{-1}\right) \in \mathscr{D} . \mathscr{D}^{Z}(G)$ is $\Sigma_{\sim}^{1}$ and closed under conjugation. In contrast to Theorem 3.3 we can only deal with Borel classes below.

Lemma 4.6. Let $\mathscr{D} \subseteq \mathscr{G}(G)^{2}$ be Borel. Then the following are equivalent:

(i) There are Borel $G$-spaces $X$ and $Y$ with $E^{X}$ and $E^{Y}$ Borel, and an invariant Borel subset $Z \subseteq X \times Y$, such that $\mathscr{D}=\mathscr{D}^{Z}(G)$.

(ii) $\mathscr{D}$ is closed under conjugation.

Proof. (i) $\Rightarrow$ (ii) is trivial.

(ii) $\Rightarrow\left(\right.$ i). Let $X=Y=\mathscr{C}(G)$. Let $Z=G^{2} \cdot \mathscr{D}$. Then clearly $Z$ is invariant and is Borel since the action of $G^{2}$ on $\mathscr{C}(G)^{2}$ has a Borel transversal $\mathscr{G}(G)^{2}$.

We suspect that in this general context the analog of Theorem 3.3 is no longer true. However, we do not know a counterexample. 
TheOREM 4.7. Let $\mathscr{D} \subseteq \mathscr{G}(G)^{2}$ be Borel and closed under conjugation. Then the following are equivalent:

(i) The map $(H, K) \mapsto H \cap K$ from $\mathscr{D}$ to $\mathscr{G}(G)$ is Borel.

(ii) For any Borel $G$-spaces $X$ and $Y$ with $E^{X}$ and $E^{Y}$ Borel and any Borel invariant $Z \subseteq X \times Y$, if $\mathscr{D}^{Z}(G) \subseteq \mathscr{D}$, then $E^{Z}$ is Borel.

(iii) There are Borel $G$-spaces $X$ and $Y$ and a Borel invariant $Z \subseteq X \times Y$ such that $\mathscr{D}^{Z}(G)=\mathscr{D}$ and $E^{X}, E^{Y}, E^{Z}$ are Borel.

(iv) There is $\alpha<\omega_{1}$ such that for $(H, K) \in \mathscr{D}, H K \in \underset{\sim}{\prod_{\alpha}^{0}}$.

Proof. (i) $\Rightarrow$ (iii) is from the preceding lemma. The other directions are similar to the proof of Theorem 4.4.

Now we are ready to present our last theorem on Borelness of the intersection operator on a general subclass of $\mathscr{G}(G)^{2}$.

THEOREM 4.8. Let $\mathscr{D} \in \mathscr{G}(G)^{2}$ be $\Sigma_{\sim}^{1}$ and closed under conjugation. Then the following are equivalent:

(i) The map $(H, K) \mapsto H \cap K$ from $\mathscr{D}$ to $\mathscr{G}(G)$ is Borel.

(ii) There are Borel $G$-spaces $X$ and $Y$ and a Borel invariant $Z \subseteq X \times Y$ such that $\mathscr{D}^{Z}(G)=\mathscr{D}$ and $E^{X}, E^{Y}, E^{Z}$ are Borel.

Proof. (ii) $\Rightarrow\left(\right.$ i). Define $\phi: Z \rightarrow \mathscr{G}(G)^{2}, \phi(x, y)=\left(G_{x}, G_{y}\right)$. Then $\phi$ is Borel and $\mathscr{D}=\mathscr{D}^{Z}(G)=\phi(Z)$. Since $E^{Z}$ is Borel, the map $(x, y) \mapsto G_{(x, y)}=G_{x} \cap G_{y}$ is Borel. By Lemma 4.2, the map $(H, K) \mapsto H \cap K$ is Borel.

(i) $\Rightarrow$ (ii). Let $\varphi: \mathscr{N} \rightarrow \mathscr{G}(G)^{2}$ be a Borel map with $\mathscr{D}=\varphi(\mathscr{N})$. Let $\varphi=\left(\varphi_{1}, \varphi_{2}\right)$. Let $s$ be a Borel selector of $\mathscr{F}(G)$. Define

$$
X=\left\{(h, x) \in G \times \mathscr{N}: h=s\left(h \varphi_{1}(x)\right)\right\}
$$

and

$$
Y=\left\{(k, y) \in G \times \mathscr{N}: k=s\left(k \varphi_{2}(y)\right)\right\} .
$$

Then $X$ and $Y$ are Borel subsets of $G \times \mathscr{N}$. Define actions of $G$ on $X$ and $Y$ respectively:

$$
\begin{aligned}
& g \cdot(h, x)=\left(s\left(g h \varphi_{1}(x)\right), x\right), \\
& g \cdot(k, y)=\left(s\left(g k \varphi_{2}(y)\right), y\right)
\end{aligned}
$$

for $g \in G,(h, x) \in X$ and $(k, y) \in Y$. Similar to Theorem 3.3, these are Borel actions of $G$ on $X$ and $Y$, respectively, with $E^{X}$ and $E^{Y}$ Borel, and $G_{(h, x)}=$ $h \varphi_{1}(x) h^{-1}, G_{(k, y)}=k \varphi_{2}(x) k^{-1}$. Now define

$$
Z=\left\{((h, x),(k, x)) \in X \times Y: h \varphi_{1}(x) \cap k \varphi_{2}(x) \neq \emptyset\right\} .
$$

Then $Z$ is a Borel subset of $X \times Y$. To see this, note that the following subset of $G \times \mathscr{N}$ is Borel:

$$
Z^{\prime}=\left\{(q, x) \in G \times \mathscr{N}: q=s\left(q\left(\varphi_{1}(x) \cap \varphi_{2}(x)\right)\right)\right\} .
$$

If we let $f: Z^{\prime} \rightarrow X \times Y$ be defined as

$$
f(q, x)=\left(\left(s\left(q \varphi_{1}(x)\right), x\right),\left(s\left(q \varphi_{2}(x)\right), x\right)\right),
$$

then $f$ is a Borel injection and $Z=f\left(Z^{\prime}\right)$. Thus $Z$ is Borel. We next verify that $Z$ is invariant. For $g \in G$ and $((h, x),(k, x)) \in Z$, let $h^{\prime}=s\left(g h \varphi_{1}(x)\right)$ and 
$k^{\prime}=s\left(g k \varphi_{2}(x)\right)$. Then $g((h, x),(k, x))=\left(\left(h^{\prime}, x\right),\left(k^{\prime}, x\right)\right)$. Since $h^{\prime} \varphi_{1}(x)=$ $g h \varphi_{1}(x)$ and $k^{\prime} \varphi_{2}(x)=g k \varphi_{2}(x)$, we have that

$$
h^{\prime} \varphi_{1}(x) \cap k^{\prime} \varphi_{2}(x)=g h \varphi_{1}(x) \cap g k \varphi_{2}(x)=g\left(h \varphi_{1}(x) \cap k \varphi_{2}(x)\right) \neq \emptyset .
$$

Thus $g \cdot((h, x),(k, x))=\left(\left(h^{\prime}, x\right),\left(k^{\prime}, x\right)\right) \in Z$.

For $((h, x),(k, x)) \in Z$, suppose $q \in h \varphi_{1}(x) \cap k \varphi_{2}(x)$. Then $h \varphi_{1}(x)=q \varphi_{1}(x)$, and

$$
h \varphi_{1}(x) h^{-1}=\left(h \varphi_{1}(x)\right)\left(h \varphi_{1}(x)\right)^{-1}=q \varphi_{1}(x) q^{-1} .
$$

Similarly, we have $k \varphi_{2}(x) k^{-1}=q \varphi_{2}(x) q^{-1}$. Since $\mathscr{D}$ is closed under conjugation,

$$
\left(G_{(h, x)}, G_{(k, x)}\right)=\left(h \varphi_{1}(x) h^{-1}, k \varphi_{2}(x) k^{-1}\right)=\left(q \varphi_{1}(x) q^{-1}, q \varphi_{2}(x) q^{-1}\right) \in \mathscr{D} .
$$

Thus $\mathscr{D}=\mathscr{D}^{Z}(G)$. Since $G_{((h, x),(k, x))}=G_{(h, x)} \cap G_{(k, x)}$, it follows from (i) that $E^{Z}$ is Borel.

Unlike the previous theorems we do not have a clause involving the Borel rank of group products in the above theorem. We do not know if the boundedness of the Borel ranks of the groups $G_{1} G_{2}$ for $\left(G_{1}, G_{2}\right) \in \mathscr{D}$ is equivalent to the Borelness of the intersection operator on $\mathscr{D}$.

§5. Countable products of countable groups. In this section we focus on the notion of relative tameness. Recall that a Polish group $G$ is relatively tame if for any Borel $G$-spaces $X$ and $Y$ with $E^{X}$ and $E^{Y}$ Borel, $E^{X \times Y}$ from the diagonal action is Borel. Following [8] we study a special class of Polish groups, namely countable products of countable groups. To emphasize the relevance of [8] we also follow the notation there. We first review some notation and facts.

A typical group in our class will be

$$
H^{\omega}=H_{0} \times H_{1} \times \cdots \times H_{n} \times \cdots,
$$

where each $H_{n}$ is a countable group. We also denote

$$
H^{n}=H_{0} \times \cdots \times H_{n-1} \text { and } H^{<\omega}=\bigcup_{n} H^{n} .
$$

A tree $S \subseteq H^{<\omega}$ is called a group tree (coset tree) on $\left(H_{n}\right)$ if $S \cap H^{n}$ is a subgroup of (coset in) $H^{n}$ for any $n \in \omega$. Let $S$ be a coset tree on $\left(H_{n}\right)$. Then for each $n \in \omega$ there is a unique subgroup $G_{n}$ of $H^{n}$ such that $S \cap H^{n}=\sigma G_{n}$ for any $\sigma \in S \cap H^{n}$. Define

$$
\alpha(S)=\bigcup_{n} G_{n} .
$$

Then it is easy to see that $\alpha(S)$ is a group tree.

Let $\mathscr{T}$ be the family of all trees on $\left(H_{n}\right)$ with the topology generated by sets of the form $\{T \in \mathscr{T}: \sigma \in T\}$ and $\{T \in \mathscr{T}: \sigma \notin T\}$ for $\sigma \in H^{<\omega}$. $\mathscr{T}$ is a Polish space. The mapping

$$
\phi:\{T \in \mathscr{T}: T \text { is pruned }\} \rightarrow \mathscr{F}\left(H^{\omega}\right)
$$

given by

$$
\phi(T)=\left\{x \in H^{\omega}: \forall n \in \omega(x\lceil n \in T)\}\right.
$$


is a Borel isomorphism. In particular the class of all pruned trees is a Borel subset of $\mathscr{T}$. We denote by $\mathscr{T}_{p}$ and $\mathcal{S}_{p}$ the families of all pruned coset trees and pruned group trees, respectively. Note that $T$ is a pruned group (coset) tree iff $\phi(T)$ is a closed subgroup (coset) of $H^{\omega}$. Hence $\mathscr{T}_{p}=\phi^{-1}\left(\mathscr{C}\left(H^{\omega}\right)\right)$ and $\mathcal{S}_{p}=\phi^{-1}\left(\mathscr{G}\left(H^{\omega}\right)\right)$, and both of them are Borel subsets of $\mathscr{T}$, hence are standard Borel spaces. The $\operatorname{map} \alpha: \mathscr{T}_{p} \rightarrow \mathcal{S}_{p}$ is Borel.

We also use the following notation. For $T \in \mathscr{T}$ and $\sigma \in T$, let $T_{\sigma}=$ $\left\{\tau: \sigma^{\wedge} \tau \in T\right\} \in \mathscr{T}$. For a well-founded tree $S$ the height of $S$, denoted $\operatorname{ht}(S)$, is just the usual well-founded rank. For a general tree $T$, the height of $T$ is defined by

$$
\operatorname{ht}(T)=\sup \left\{\operatorname{ht}\left(T_{\sigma}\right): T_{\sigma} \text { is well-founded }\right\} .
$$

The following theorems characterize relative tameness in terms of height of coset trees and group trees. The results are certainly motivated by those in [8].

THEOREM 5.1. $H^{\omega}$ is relatively tame iff there is $\beta<\omega_{1}$ such that, for any two pruned coset trees $S$ and $T$, if $S \cap T$ is well-founded, then $\operatorname{ht}(S \cap T)<\beta$.

Proof. $(\Rightarrow)$ Define an $H^{\omega}$ action on $\mathscr{T}_{p}$ by

$$
x \cdot S=\bigcup_{m \in \omega}\left(x\lceil m)\left(S \cap H^{m}\right) .\right.
$$

Then $H_{\alpha(S), S}^{\omega}=\phi(S)$ and

$$
H^{\omega} \cdot S=H^{\omega} \cdot T \Longleftrightarrow \alpha(S)=\alpha(T) .
$$

It follows easily that $\mathscr{T}_{p}$ is a Borel $H^{\omega}$-space and $E^{\mathscr{T}_{p}}$ is Borel.

Now if $H$ is relatively tame, then $E^{\mathscr{F}_{p}^{2}}$ is Borel too. Thus the set

$$
\mathscr{A}=\{(S, T):(S, T) \text { and }(\alpha(S), \alpha(T)) \text { are not in the same orbit }\}
$$

is also Borel. However, for any $S, T \in \mathscr{T}_{p}$,

$(S, T)$ and $(\alpha(S), \alpha(T))$ are in the same orbit

$$
\begin{aligned}
& \Longleftrightarrow H_{\alpha(S), S}^{\omega} \cap H_{\alpha(T), T}^{\omega}=\phi(S) \cap \phi(T) \neq \emptyset \\
& \Longleftrightarrow S \cap T \text { is ill-founded. }
\end{aligned}
$$

Since $(S, T) \mapsto S \cap T$ is continuous from $\mathscr{T}_{p}^{2}$ to $\mathscr{T}$, we have that

$$
\{S \cap T:(S, T) \in \mathscr{A}\}=\left\{S \cap T: S, T \in \mathscr{T}_{p} \text { and } S \cap T \text { is well-founded }\right\}
$$

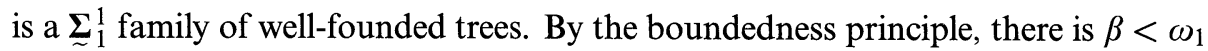
such that, for any $S, T \in \mathscr{T}_{p}$, if $S \cap T$ is well-founded, then $\operatorname{ht}(S \cap T)<\beta$.

$(\Leftarrow)$ Let $X$ and $Y$ be Borel $H^{\omega}$-spaces with $E^{X}$ and $E^{Y}$ Borel. For any $x, u \in X$, $y, v \in Y$, let $S(x, u)=\phi^{-1}\left(H_{x, u}^{\omega}\right)$ and $T(y, v)=\phi^{-1}\left(H_{y, v}^{\omega}\right)$. Then $(x, u) \mapsto S(x, u)$ and $(y, v) \mapsto T(y, v)$ are Borel maps into $\mathscr{T}_{p}$. We have that

$$
\begin{aligned}
H^{\omega} \cdot(x, y)=H^{\omega} \cdot(u, v) & \Longleftrightarrow H_{x, u}^{\omega} \cap H_{y, v}^{\omega} \neq \emptyset \\
& \Longleftrightarrow S(x, u) \cap T(y, v) \text { is ill-founded. }
\end{aligned}
$$

Since $\{T \in \mathscr{T}: T$ is well-founded and ht $(T)<\beta\}$ is Borel, $E^{X \times Y}$ is Borel.

THEOREM 5.2. $H^{\omega}$ is relatively tame iff there is $\beta<\omega_{1}$ such that, for any two pruned group trees $S$ and $T, \operatorname{ht}(S \cap T)<\beta$. 
Proof. $(\Rightarrow)$ Since $H^{\omega}$ is relatively tame the map $(G, K) \mapsto G \cap K$ from $\mathscr{G}\left(H^{\omega}\right)^{2}$ to $\mathscr{G}\left(H^{\omega}\right)$ is Borel. For any $\sigma \in H^{<\omega}$, let $U_{\sigma}=\left\{x \in H^{\omega}: \sigma \subseteq x\right\}$. Then

$$
\left\{(G, K) \in \mathscr{G}\left(H^{\omega}\right)^{2}: G \cap K \cap U_{\sigma} \neq \emptyset\right\}
$$

is Borel for any $\sigma \in H^{<\omega}$. Let $S=\phi^{-1}(G)$ and $T=\phi^{-1}(K)$. Then $S, T \in \mathcal{S}_{p}$ and

$$
G \cap K \cap U_{\sigma} \neq \emptyset \Longleftrightarrow(S \cap T)_{\sigma} \text { is ill-founded. }
$$

Therefore

$$
\left\{(S, T) \in \mathcal{S}_{p}^{2}:(S \cap T)_{\sigma} \text { is well-founded }\right\}
$$

is Borel. By the boundedness principle, there is $\beta_{\sigma}<\omega_{1}$ such that, whenever $(S \cap T)_{\sigma}$ is well-founded, $\operatorname{ht}\left((S \cap T)_{\sigma}\right)<\beta_{\sigma}$. Put $\beta=\sup \left\{\beta_{\sigma}: \sigma \in H^{<\omega}\right\}$. Then ht $(S \cap T)<\beta$ for any $S, T \in \mathcal{S}_{p}$.

$(\Leftarrow)$ By reversing the above argument the converse can be similarly proved.

For the rest of this section all groups will be abelian. We will use + for the group operation and 0 for the group identity.

Recall the following definition from [8]. An abelian countable group $H$ is said to be manageable if there exist two decreasing sequences of subgroups $\left(G_{n}^{0}\right),\left(G_{n}^{1}\right)$ with $\bigcap_{n} G_{n}^{i}=\{0\}$ for $i=0,1$, and a homomorphism $\varphi: H \times H \rightarrow H$ such that $\varphi\left[G_{n}^{0} \times G_{n}^{1}\right]=H$ for any $n \in \omega$. It was proved in [8] that $\mathbb{Z}$ and $\oplus_{\omega} \mathbb{Z}(p)$, where $p$ is prime, are manageable. For $\mathbb{Z}$, put $G_{n}^{0}=\left\{2^{n} k: k \in \mathbb{Z}\right\}, G_{n}^{1}=\left\{3^{n} k: k \in \mathbb{Z}\right\}$ and $\varphi(m, l)=m+l$. As to $\oplus_{\omega} \mathbb{Z}(p)$, let $\langle m, n\rangle$ be an enumeration of $\omega \times \omega$ and put $G_{n}^{0}=\{0\}, G_{n}^{1}=\left\{x \in \oplus_{\omega} \mathbb{Z}(p): x\lceil n=(0,0, \ldots, 0)\}\right.$ and $\varphi(0, x)=$ $\left(\sum_{m} x(\langle m, n\rangle)\right)$.

LEMMA 5.3. Let $H$ be a countable abelian group. If $H$ is manageable, then for any $\beta<\omega_{1}$, there are pruned group trees $S_{\beta}, T_{\beta} \subseteq H^{<\omega}$ such that $\mathrm{ht}\left(S_{\beta} \cap T_{\beta}\right) \geq \beta$. Furthermore, we can find such $S_{\beta}, T_{\beta}$ that the only infinite branch in $S_{\beta} \cap T_{\beta}$ is $(0,0, \ldots)$.

PROoF. Let $\left(G_{n}^{0}\right),\left(G_{n}^{1}\right)$ witness manageability of $H$. We will produce group trees $S_{\beta}$ and $T_{\beta}$ for all $\beta<\omega_{1}$ with the following properties:

(a) $(0,0, \ldots)$ is the unique infinite branch of $S_{\beta} \cap T_{\beta}$;

(b) if $\beta$ is a successor, then

$$
\forall h \in H\left(h \neq 0 \Rightarrow \mathrm{ht}\left(S_{\beta} \cap T_{\beta}\right)_{h} \geq \beta\right) ;
$$

in particular $S_{\beta} \cap T_{\beta} \cap H=H$;

(c) if $\beta$ is a limit, then $\forall \gamma<\beta \exists n \in \omega$

$$
\forall\left(h_{0}, h_{1}\right) \in G_{n}^{0} \times G_{n}^{1}\left(\left(h_{0}, h_{1}\right) \neq(0,0) \Rightarrow \mathrm{ht}\left(S_{\beta} \cap T_{\beta}\right)_{\left(h_{0}, h_{1}\right)} \geq \gamma\right) .
$$

We define $S_{\beta}$ and $T_{\beta}$ by transfinite induction on $\beta<\omega_{1}$. For $\beta=0$, put

$$
S_{0}=\{(h, h, h, \ldots, h): h \in H\} \text { and } T_{0}=\{(h, 0,0, \ldots, 0): h \in H\} .
$$

Then obviously $S_{0}$ and $T_{0}$ are pruned and $S_{0} \cap T_{0}=H \cup\{(0,0, \ldots, 0)\}$. If $\beta=\gamma+1$ and $\gamma$ is a successor, put

$$
\begin{aligned}
& S_{\beta}=\{\emptyset\} \cup H \cup\left\{\sigma(0)^{\frown} \sigma: \sigma \in S_{\gamma}, \sigma \neq \emptyset\right\}, \\
& T_{\beta}=\{\emptyset\} \cup H \cup\left\{\sigma(0)^{\frown} \sigma: \sigma \in T_{\gamma}, \sigma \neq \emptyset\right\} .
\end{aligned}
$$


Then $S_{\beta}$ and $T_{\beta}$ are pruned since $S_{\gamma}$ and $T_{\gamma}$ are, and since $S_{\gamma} \cap H=T_{\gamma} \cap H=H$ by the inductive hypothesis. We also have

$$
S_{\beta} \cap T_{\beta}=\{\emptyset\} \cup H \cup\left\{\sigma(0)^{\frown} \sigma: \sigma \in S_{\gamma} \cap T_{\gamma}, \sigma \neq \emptyset\right\}
$$

and thus (b) is satisfied by the inductive hypothesis.

We assume $\beta<\omega_{1}$ is a limit ordinal. Fix an increasing sequence of successors $\gamma_{n} \rightarrow \beta, n \in \omega$. Fix also a partition $\left(X_{n}\right)$ of $\omega$ into infinite sets. Denote $a_{n}=\min X_{n}$. For simplicity assume that $\left(a_{n}\right)$ is an increasing sequence. For any $m \in \omega$, let $k_{m}=\max \left\{n: a_{n}<m\right\}$. For $i=0,1$ define group trees $S_{\beta}^{i}$ and $T_{\beta}^{i}$ by letting, for $m>0, S_{\beta}^{i} \cap H^{m+1}$ be

$$
\left\{h^{\frown} \sigma: \forall n \leq k_{m}\left(\sigma \mid X_{n} \in S_{\gamma_{n}} \wedge \sigma\left(a_{n}\right) \in G_{n}^{i}\right) \wedge h-\sum_{n=0}^{k_{m}} \sigma\left(a_{n}\right) \in G_{k_{m}+1}^{i}\right\} ;
$$

and similarly letting $T_{\beta}^{i} \cap H^{m+1}$ be

$$
\left\{h^{\frown} \sigma: \forall n \leq k_{m}\left(\sigma \mid X_{n} \in T_{\gamma_{n}} \wedge \sigma\left(a_{n}\right) \in G_{n}^{i}\right) \wedge h-\sum_{n=0}^{k_{m}} \sigma\left(a_{n}\right) \in G_{k_{m}+1}^{i}\right\} .
$$

Let $S_{\beta}^{i} \cap H=T_{\beta}^{i} \cap H=G_{0}^{i}$. It is easy to see that $S_{\beta}^{i} \cap H^{m+1}$ and $T_{\beta}^{i} \cap H^{m+1}$ are groups for all $m \in \omega$. To see that $S_{\beta}^{i}$ and $T_{\beta}^{i}$ are trees, let $m^{\prime}<m, h^{\frown} \sigma \in S_{\beta}^{i} \cap H^{m+1}$ (similarly for $\left.T_{\beta}^{i} \cap H^{m+1}\right)$ and $h^{\frown} \sigma^{\prime}=h^{\frown} \sigma \uparrow\left(m^{\prime}+1\right)$. Then it follows from

$$
\forall n \leq k_{m}\left(\sigma \mid X_{n} \in S_{\gamma_{n}} \wedge \sigma\left(a_{n}\right) \in G_{n}^{i}\right)
$$

that

$$
\forall n \leq k_{m^{\prime}} \leq k_{m}\left(\sigma^{\prime} \mid X_{n} \in S_{\gamma_{n}} \wedge \sigma\left(a_{n}\right) \in G_{n}^{i}\right)
$$

Also from

$$
h-\sum_{n=0}^{k_{m}} \sigma\left(a_{n}\right) \in G_{k_{m}+1}^{i}
$$

it follows that

$$
h-\sum_{n=0}^{k_{m^{\prime}}} \sigma\left(a_{n}\right) \in \sum_{n=k_{m^{\prime}}+1}^{k_{m}} \sigma\left(a_{n}\right)+G_{k_{m}+1}^{i} \subseteq \sum_{n=k_{m^{\prime}}+1}^{k_{m}+1} G_{n}^{i} \subseteq G_{k_{m^{\prime}}+1}^{i}
$$

since the sequence $\left(G_{n}^{i}\right)$ is decreasing. Thus $h^{\curvearrowleft} \sigma^{\prime} \in S_{\beta}^{i}$.

We claim that $S_{\beta}^{i}$ and $T_{\beta}^{i}$ are pruned. By inductive hypothesis all $S_{\gamma_{n}}$ and $T_{\gamma_{n}}$ are pruned. Fix an arbitrary $h^{\curvearrowleft} \sigma \in S_{\beta}^{i}$ and let $m=\operatorname{lh}(\sigma)$. For any $n \leq k_{m}$, let $x_{n}$ be an infinite branch of $S_{\gamma_{n}}$ such that $\sigma\left\lceil X_{n} \subseteq x_{n}\right.$. Note that $h^{\prime}=h-\sum_{n=0}^{k_{m}} \sigma\left(a_{n}\right) \in G_{k_{m}+1}^{i}$. Since $\gamma_{k_{m}+1}$ is a successor, we can find an $x_{k_{m}+1} \in S_{\gamma_{k_{m}+1}}$ such that $h^{\prime}=x_{k_{m}+1}(0)$. Let $x$ be given by

$$
\forall n \leq k_{m}+1\left(x \uparrow X_{n}=x_{n}\right) \wedge \forall n>k_{m}+1\left(x \uparrow X_{n}=(0,0, \ldots)\right) .
$$

Clearly $x\left\lceil m=\sigma\right.$. In fact $h^{\curvearrowleft} x$ is an infinite branch of $S_{\beta}^{i}$. We have thus shown that $S_{\beta}^{i}$ is pruned. Similarly $T_{\beta}^{i}$ is also pruned. 
Next we claim that the only infinite branch of $S_{\beta}^{i} \cap T_{\beta}^{i}$ is $(0,0, \ldots)$. Suppose $h^{\frown} x$ is an infinite branch of $S_{\beta}^{i} \cap T_{\beta}^{i}$. Then $x \uparrow X_{n}$ is an infinite branch of $S_{\gamma_{n}} \cap T_{\gamma_{n}}$ for all $n \in \omega$, and therefore by the inductive hypothesis $x\left\lceil X_{n}=(0,0, \ldots)\right.$ for all $n \in \omega$. Hence $x=(0,0, \ldots)$. Now for any $m \in \omega, h^{\frown}\left(x\lceil m) \in S_{\beta}^{i} \cap T_{\beta}^{i} \cap H^{m+1}\right.$, so $h \in G_{k_{m}+1}^{i}$. It follows that $h=0$ and the only infinite branch of $S_{\beta}^{i} \cap T_{\beta}^{i}$ is $(0,0, \ldots)$.

The following computation will be useful for the verification of (c). If $h \in G_{n}^{i}$, $h \neq 0$, consider $\sigma=(0,0, \ldots, 0, h) \in H^{a_{n}+1}$. By our construction $h^{\frown} \sigma \in S_{\beta}^{i} \cap T_{\beta}^{i}$ and ht $\left(S_{\beta}^{i} \cap T_{\beta}^{i}\right)_{h \frown \sigma} \geq \operatorname{ht}\left(S_{\gamma_{n}} \cap T_{\gamma_{n}}\right)_{h} \geq \gamma_{n}$.

Finally define

$$
S_{\beta}=\left\{\sigma \in H^{<\omega}: \sigma \uparrow\{2 k: k \in \omega\} \in S_{\beta}^{0} \wedge \sigma\left\lceil\{2 k+1: k \in \omega\} \in S_{\beta}^{1}\right\}\right.
$$

and similarly

$$
T_{\beta}=\left\{\sigma \in H^{<\omega}: \sigma\left\lceil\{2 k: k \in \omega\} \in T_{\beta}^{0} \wedge \sigma\left\lceil\{2 k+1: k \in \omega\} \in T_{\beta}^{1}\right\} .\right.\right.
$$

Then $S_{\beta}$ and $T_{\beta}$ are pruned group trees. The requirements (a) and (c) follow from the properties of $S_{\beta}^{i}$ and $T_{\beta}^{i}$ noted above.

The remaining case in our inductive definition is when $\beta=\gamma+1$ and $\gamma$ is a limit. In this case let $S_{\beta}$ be the tree generated by $\left\{\varphi(\sigma(0), \sigma(1))^{\sim} \sigma: \sigma \in S_{\gamma}, \operatorname{lh}(\sigma) \geq 2\right\}$ and $T_{\beta}$ the tree generated by $\left\{\varphi(\sigma(0), \sigma(1)) \frown \sigma: \sigma \in T_{\gamma}, \operatorname{lh}(\sigma) \geq 2\right\}$. Then $S_{\beta}$ and $T_{\beta}$ are pruned group trees. Requirement (b) holds by manageability and the inductive hypothesis on $S_{\gamma}$ and $T_{\gamma}$.

COROllary 5.4. Let $H$ be a countable abelian group. If $H$ is manageable, then $\left\{(G, K) \in \mathscr{G}\left(H^{\omega}\right): G \cap K=\{0\}\right\}$ is not Borel, hence $H^{\omega}$ is not relatively tame.

Proof. Assume $H$ is manageable and $\left\{(G, K) \in \mathscr{G}\left(H^{\omega}\right): G \cap K=\{0\}\right\}$ is Borel. Using the Borel isomorphism $\phi$ between $\mathcal{S}_{p}$ and $\mathscr{G}\left(H^{\omega}\right)$, we get that

$$
\mathscr{A}=\left\{(S, T) \in \mathcal{S}_{p}^{2}: S \cap T \text { has only one infinite branch }(0,0, \ldots)\right\}
$$

is Borel. By the boundedness principle, there is $\beta<\omega_{1}$ such that $\operatorname{ht}(S \cap T)<\beta$ for all $(S, T) \in \mathscr{A}$. This contradicts the preceding lemma.

THEOREM 5.5. Let $\left(H_{n}\right)$ be a sequence of countable abelian groups. Then $H^{\omega}$ is relatively tame iff $H^{\omega}$ is tame.

Proof. The $(\Leftarrow)$ direction is trivial. We only argue for the $(\Rightarrow)$ direction. If $H^{\omega}$ is not tame, then by Lemmas 8 and 9 of [8], there are infinitely many $n \in \omega$ and a prime number $p$ such that either $H_{n}$ is torsion-free or there is a surjective homomorphism mapping a subgroup of $H_{n}$ onto $\oplus_{\omega} \mathbb{Z}(p)$. It follows that either there is a closed subgroup of $H^{\omega}$ isomorphic to $\mathbb{Z}^{\omega}$ or there is a closed subgroup of $H^{\omega}$ with a continuous homomorphism from it onto $\left(\oplus_{\omega} \mathbb{Z}(p)\right)^{\omega}$. In either case $H^{\omega}$ is not relatively tame by the preceding corollary.

We say that a Polish group $G$ is $\sigma$-relatively tame if for any infinite sequence $\left(X_{n}\right)$ of Borel $G$-spaces with each $E^{X_{n}}$ Borel, $E \prod X_{n}$ is Borel. The concept of $\sigma$-relative tameness implies relative tameness but is weaker than that of tameness. Thus $H^{\omega}$ is $\sigma$-relatively tame iff it is relative tame. In general we do not have any examples to distinguish these three concepts. We conjecture that there are such examples in the class of countable products of countable groups. 
§6. Logic actions. In this last section we make some observations and remarks on logic actions of the infinite symmetric group $S_{\infty}$. Basic notation and facts can be found in [1]. Borel equivalence relations induced by $S_{\infty}$-actions were also investigated in [2] and [3]. The reader can find most of the well known facts mentioned below in one of these sources.

Let $L$ be a countable relational language and $\operatorname{Mod}(L)$ be the space of all countable $L$-structures with underlying universe $\omega$. The logic action of $S_{\infty}$ on $\operatorname{Mod}(L) \operatorname{makes}$ it a Polish $S_{\infty}$-space. The orbit equivalence relation $E^{\operatorname{Mod}(L)}$ is the isomorphism relation on $\operatorname{Mod}(L)$, which we denote by $\cong_{L}$. Let $\sigma \in L_{\omega_{1} \omega}$ be a sentence. Then $\operatorname{Mod}(\sigma) \subseteq \operatorname{Mod}(L)$ is an invariant Borel subset. We also denote $\cong_{L}\lceil\operatorname{Mod}(\sigma)$ by $\cong_{\sigma}$.

It is well known that $\cong_{\sigma}$ is Borel iff there is a uniform bound on the Scott ranks of models in $\operatorname{Mod}(\sigma)$, i.e., there is $\alpha<\omega_{1}$ such that $\operatorname{sr}(M)<\alpha$ for all $M \in \operatorname{Mod}(L)$. The stabilizer map, on the other hand, is exactly $M \mapsto \operatorname{Aut}(M)$, where $\operatorname{Aut}(M)$ is the automorphism group of $M$.

Diagonal actions of $S_{\infty}$ can be interpreted in the following sense. Let $L$ and $L^{\prime}$ be disjoint relational languages, $\sigma \in L_{\omega_{1} \omega}$ and $\sigma^{\prime} \in L_{\omega_{1} \omega}^{\prime}$ be sentences and $\tau \in\left(L \cup L^{\prime}\right)_{\omega_{1} \omega}$ be a sentence in the expansion. Assume that $\models \tau \rightarrow\left(\sigma \wedge \sigma^{\prime}\right)$ and that $\cong{ }_{\sigma}$ and $\cong_{\sigma^{\prime}}$ are Borel. Then $\operatorname{Mod}(\tau)$ is essentially an invariant Borel subset of $\operatorname{Mod}(\sigma) \times \operatorname{Mod}\left(\sigma^{\prime}\right) \subseteq \operatorname{Mod}(L) \times \operatorname{Mod}\left(L^{\prime}\right)$, where the action of $S_{\infty}$ on the product space is the diagonal action. By our Theorem $4.7 \cong_{\tau}$ is Borel iff there is $\alpha<\omega_{1}$ such that $\operatorname{Aut}\left(M_{L}\right) \operatorname{Aut}\left(M_{L^{\prime}}\right) \in \prod_{\sim}^{0}$ for all $M \in \operatorname{Mod}(\tau)$, where $M_{L}$ and $M_{L^{\prime}}$ are the reducts of $M$ to $L$ and $L^{\prime}$, respectively. In particular this holds if $\operatorname{Aut}\left(M_{L}\right)$ is locally compact for all $M \in \operatorname{Mod}(\tau)$.

We now turn to a concrete example of $S_{\infty}$-action and determine the complexity of the orbit equivalence relations up to Borel reducibility.

THeOREM 6.1. Let $E_{n}, n \in \omega \cup\{\omega\}$, be the orbit equivalence relation induced by the conjugacy action of $S_{\infty}$ on $S_{\infty}^{n}$. Then for each $1<n \leq \omega, E_{n}$ is Borel bireducible to $F_{2}$, the identity of countable sets of reals.

Proof. Clearly for $m<n \leq \omega, E_{m} \leq_{B} E_{n}$. Thus it suffices to show that $E_{\omega} \leq_{B} F_{2}$ and $F_{2} \leq_{B} E_{2}$.

Given $\vec{f}=\left(f_{n}\right) \in S_{\infty}^{\omega}$, define the structure $M_{\vec{f}}=\left(\omega,\left(f_{n}\right)\right)$. Then $E_{\omega}$ is the isomorphism relation on the class of structures $\left\{M_{\vec{f}}: \vec{f} \in S_{\infty}^{\omega}\right\}$. We first analyze the structure $M_{\vec{f}}$. Each $M_{\vec{f}}$ can be decomposed into a disjoint union of substructures. For each $a \in \omega$, let $C_{a}$ be the smallest subset of $\omega$ containing $a$ and closed under all $f_{n}$ and $f_{n}^{-1}$; call $C_{a}$ the component of $a$. Then $\omega$ is decomposed into at most countably many components, and each component induces a substructure.

Let $F_{\infty}$ be the free group generated by the alphabet $\left\{\sigma_{n}: n \in \omega\right\}$ with symbols $\sigma_{n}$. For $u \in F_{\infty}$ and given $\vec{f} \in S_{\infty}^{\omega}$ let $u(\vec{f})$ be the permutation resulting from replacing each occurrence of $\sigma_{n}$ by $f_{n}$. Consider a pointed structure $N_{\vec{f}, a}=\left(C_{a}, \vec{f}\left\lceil C_{a}, a\right)\right.$ and define a relation $R_{\vec{f}, a}$ on $F_{\infty}$ by $R_{\vec{f}, a}(u, v)$ iff $u(\vec{f})(a)=v(\vec{f})(a)$. Then $R_{\vec{f}, a}$ is an equivalence relation and the isomorphism type of $N_{\vec{f}, a}$ is completely determined by $R_{\vec{f}, a}$. More specifically, $N_{\vec{f}, a} \cong N_{\vec{g}, b}$ iff $R_{\vec{f}, a}=R_{\vec{g}, b}$. Since $R_{\vec{f}, a}$ can be coded by a subset of $\omega$, this shows that the isomorphism relation between pointed components is smooth. We let $r(\vec{f}, a) \in 2^{\omega}$ be the real coding $R_{\vec{f}, a}$. 
Now for each $a \in \omega$ and $u \in F_{\infty}$ let $\theta_{u}(\vec{f}, a)=r(\vec{f}, u(\vec{f})(a))$. Note that $C_{u(\vec{f})(a)}=C_{a}$. We have thus obtained countably many Borel functions $\theta_{u}: S_{\infty}^{\omega} \times \omega \rightarrow 2^{\omega}$ such that for any $\vec{f}, \vec{g} \in S_{\infty}^{\omega}$ and $a, b \in \omega,\left(C_{a}, \vec{f}\left\lceil C_{a}\right) \cong\right.$ $\left(C_{b}, \vec{g} \mid C_{b}\right)$ iff $\left\{\theta_{u}(\vec{f}, a): u \in F_{\infty}\right\}=\left\{\theta_{u}(\vec{g}, b): u \in F_{\infty}\right\}$ iff $\left\{\theta_{u}(\vec{f}, a): u \in F_{\infty}\right\} \cap$ $\left\{\theta_{u}(\vec{g}, b): u \in F_{\infty}\right\} \neq \emptyset$.

For $\vec{f} \in S_{\infty}^{\omega}$ and $a \in \omega$, let $n(\vec{f}, a)$ be the number of distinct components $C_{b}$ in $M_{\vec{f}}$ so that $C_{b} \cong C_{a}$, if this number is finite; and let $n(\vec{f}, a)=0$ if this number is infinite. It is easy to check that $n: S_{\infty}^{\omega} \times \omega \rightarrow \omega$ is a Borel function.

Finally fix a continuous bijection $\langle\cdot, \cdot\rangle: 2^{\omega} \times \omega \rightarrow 2^{\omega}$. For $\vec{f} \in S_{\infty}^{\omega}$ define $\rho(\vec{f})$ to be the set $\left\{\left\langle\theta_{u}(\vec{f}, a), n(\vec{f}, a)\right\rangle: a \in \omega, u \in F_{\infty}\right\} . \rho(\vec{f})$ is a countable set of reals. Now $\rho(\vec{f})$ codes the isomorphism types of all components of $M_{\vec{f}}$ as well as the number of isomorphic components within $M_{\vec{f}}$. Thus $M_{\vec{f}} \cong M_{\vec{g}}$ iff $\rho(\vec{f})=\rho(\vec{g})$. This completes the proof that $E_{\omega} \leq_{B} F_{2}$.

It remains to show that $F_{2} \leq_{B} E_{2}$. If $A \subseteq \omega$ is infinite, let $n_{A}$ denote the $n$-th element of $A$; thus $A$ can be enumerated in the increasing order as $0_{A}, 1_{A}, \ldots$ For any permutation $f \in S_{\infty}$ and infinite $A \subseteq \omega$, let $f_{A}: A \rightarrow A$ be the permutation given by

$$
f_{A}\left(n_{A}\right)=m_{A} \Longleftrightarrow f(n)=m .
$$

Arbitrarily fix a continuous injection $x \mapsto f^{x}$ from $2^{\omega}$ into $S_{\infty}$. For instance, fixing a recursive bijection $\langle\cdot, \cdot\rangle: \omega \times \omega \rightarrow \omega$, we can let $f^{x}$ contain a cycle

$$
(\langle 0, n\rangle,\langle 1, n\rangle, \ldots,\langle n+1, n\rangle)
$$

for each $n \in x$, and no other cycles. Thus for any $n \in \omega, f^{x}$ contains a cycle of length $n+2$ iff $n \in x$ iff $x(n)=1$.

Finally fix a partition of $\omega$ into a doubly indexed family of disjoint infinite sets $A_{k, l}$ for $k, l \in \omega$. For instance, we can let $A_{k, l}=\{\langle p,\langle k, l\rangle\rangle: p \in \omega\}$. Then for each $x=\left(x_{0}, x_{1}, \ldots, x_{k}, \ldots\right) \in 2^{\omega \times \omega}$ where each $x_{k} \in 2^{\omega}$, define permutations

$$
\pi_{1}(x)=\bigcup_{k, l} f_{A_{k, l}}^{x_{k}}
$$

and

$$
\pi_{2}(x)=\bigcup_{k, l} g_{A_{k, l}},
$$

where $g \in S_{\infty}$ is the fixed permutation $(\ldots, 4,2,0,1,3, \ldots)$. We claim that for $x, x^{\prime} \in 2^{\omega \times \omega},\left\{x_{0}, x_{1}, \ldots,\right\}=\left\{x_{0}^{\prime}, x_{1}^{\prime}, \ldots\right\}$ iff $\left(\pi_{1}(x), \pi_{2}(x)\right)$ and $\left(\pi_{1}\left(x^{\prime}\right), \pi_{2}\left(x^{\prime}\right)\right)$ are conjugate. To see this, just note that $\pi_{2}(x)$ codes the partition $\left\{A_{k, l}\right\}$ of $\omega$, and within each $A_{k, l}, \pi_{1}(x)$ codes the permutation $f^{x_{k}}$, and hence in turn codes the real $x_{k}$. Thus each real $x_{k}$ is coded infinitely many times by the cycle structure of $\left(\pi_{1}(x), \pi_{2}(x)\right)$, and therefore only the set of $\left\{x_{0}, x_{1}, \ldots, x_{k}, \ldots\right\}$ (rather than the sequence itself) is coded by the cycle structure. This finishes the proof of the theorem.

It is well known and implicit in the above proof that the conjugacy equivalence relation on $S_{\infty}$ is smooth. The above theorem gives a non-trivial example of the diagonal equivalence relation being Borel. It is easy to see that for any permutation $f \in S_{\infty}$, the stabilizer group $G_{f}$ can be expressed as the product of a number of 
wreath products (c.f. [5] for definition and notation); in particular, $G_{f}$ is isomorphic to a group of the form

$$
\prod_{n} S_{m_{n}} \ltimes(\mathbb{Z} /(n))^{m_{n}}
$$

where $m_{n} \leq \omega$. Let $\mathcal{S}=\left\{G_{f}: f \in S_{\infty}\right\}$. Then by our Theorem 4.4 the intersection operator on $\mathcal{S}$ is Borel. This Borelness is obscure when the intersection operation is considered directly. Moreover, from the above proof we can obtain that for $\left(f_{1}, f_{2}\right) \in S_{\infty}^{2}$ the stabilizer $G_{\left(f_{1}, f_{2}\right)}=G_{f_{1}} \cap G_{f_{2}}$ is also a product of a number of wreath products. More specifically, $G_{\left(f_{1}, f_{2}\right)}$ is isomorphic to a group of the form

$$
\prod_{n} S_{m_{n}} \ltimes G_{n}^{m_{n}}
$$

where $G_{n}$ is a countable group with 2 generators. It is clear that the groups $G_{n}$ can range over all 2-generator groups. In general, given a group of the form (2) with arbitrary countable $G_{n}$, by considering the Cayley graphs we may construct an at most countable sequence of permutations $\vec{f}$ so that the given group is isomorphic to the stabilizer of $\vec{f}$ in the diagonal action. It follows that one can rewrite the group as an intersection of at most countably many groups of form (1). Moreover, if there is $k \in \omega$ such that all $G_{n}$ 's are $k$-generator groups, then we need only $k$ many functions in $\vec{f}$ and thus $k$ many groups of form (1) in the intersection.

\section{REFERENCES}

[1] H. BECKER and A. S. KECHRIS, The descriptive set theory of Polish group actions, London Mathematical Society Lecture Notes Series, vol. 232, Cambridge University Press, 1996.

[2] G. HJORTH and A. S. KeCHRIS, Borel equivalence relations and classifications of countable models, Annals of Pure and Applied Logic, vol. 82 (1996), pp. 221-272.

[3] G. HJorth, A. S. KeCHRIS, and A. LOUvEAU, Borel equivalence relations induced by actions of the symmetric group, Annals of Pure and Applied Logic, vol. 92 (1998), pp. 63-112.

[4] A. S. KeCHRIS, Classical descriptive set theory, Graduate Texts in Mathematics, vol. 156, SpringerVerlag, 1995.

[5] D. J. S. Robinson, A course in the theory of groups, Graduate Texts in Mathematics, vol. 80, Springer, 1996.

[6] R. SAMI, Polish group actions and the Vaught conjecture, Transactions of the American Mathematical Society, vol. 341 (1994), pp. 335-353.

[7] Y. S. SAmollenko, Spectral theory of families of self-adjoint operators, Mathematics and Its Applications (Soviet Series), vol. 57, Kluwer Academic Publishers, 1991.

[8] S. SOLECKI, Equivalence relations induced by actions of Polish groups, Transactions of the American Mathematical Society, vol. 347 (1995), pp. 4765-4777.

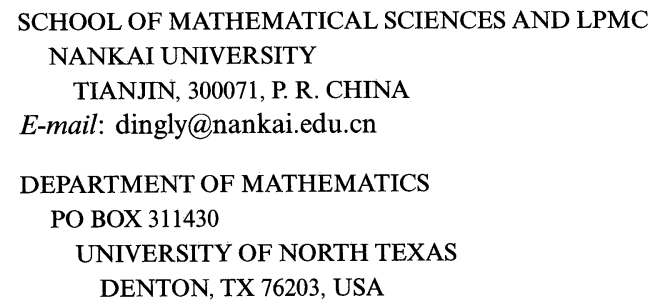

E-mail: sgao@unt.edu 